\title{
Review
}

\section{Assessment of Vitamin A Supplementation Practices in Countries of the Eastern Mediterranean Region: Evidence to Implementation}

\author{
Farah SAAD ${ }^{1}$, Lisa Rogers ${ }^{2}$, Radhouene DogGUI ${ }^{3,4}$ and Ayoub AL-JAWALDEH ${ }^{1, *}$ \\ ${ }^{1}$ Regional Office for the Eastern Mediterranean (EMRO), World Health Organization (WHO), \\ Monazamet El Seha El Alamia Str, Extension of Abdel Razak El Sanhouri Street, \\ P.O. Box 7608, Nasr City, Cairo 11371, Egypt \\ ${ }^{2}$ Department of Nutrition for Health and Development, World Health Organization, Geneva, Switzerland \\ ${ }^{3}$ Department of Family Medicine, Université de Sherbrooke, Sherbrooke, Québec, Canada \\ ${ }^{4}$ Centre de Formation Médicale du Nouveau-Brunswick, Moncton, New Brunswick, Canada
}

(Received June 21, 2020)

\begin{abstract}
Summary Vitamin A is an essential nutrient necessary for human growth and development, with critical roles in vision, immune function reproduction and maintenance of epithelial cellular integrity. Inadequate intake of vitamin A places populations at risk of developing diseases associated with vitamin A deficiency (VAD). VAD is highly prevalent across the Eastern Mediterranean Region (EMR) in children under $5 \mathrm{y}$ and women of childbearing age. Therefore, infants and young children, pregnant women and postpartum women are commonly targeted by supplementation programs. Although, vitamin A supplementation has been shown to decrease preventable childhood diseases and deaths related to VAD, supplementation of vitamin A has been greatly misused in several countries within the EMR raising concern around the process of supplementing the target population. Countries across the EMR have reported different supplementation practices depending on the income level of the country, the availability of vitamin A and the prevalence rates of VAD. Although some countries had higher supplementation rates than others, the concern lies in the middle-income countries and their supplementation practices. Some of the countries across the region do not follow the World Health Organization's (WHO) guidelines for vitamin A supplementation for the recommended age groups. The objective of this study is to assess the vitamin A supplementation practices across the countries in the EMR, determine the gaps in the supplementation practices and the issue with supplementing to healthy populations where VAD is not a public health concern, and provide recommendations for proper vitamin A supplementation within the region.
\end{abstract}

Key Words Retinol deficiency, supplementation, fortification programs, supplementation, Eastern Mediterranean Region.

Vitamin A is an essential nutrient necessary for human growth and development, with critical roles in vision, immune function, reproduction, and maintenance of epithelial cellular integrity (1). Essential nutrients cannot be synthesized by the body and must therefore be provided by the diet. Vitamin A may be consumed as either preformed vitamin A or provitamin A carotenoids. Preformed vitamin A is found in animal source foods such as human and animal milk and other dairy products; glandular meats, including liver; fish liver oils; and egg yolks. Provitamin A carotenoids are found in plant sources such as green leafy vegetables, yellow vegetables, yellow and orange non-citrus fruits, red palm oil, and other indigenous plants like palm fruit found in Brazil (1). Although provitamin A carotenoids have lower amounts of biologically available vitamin A, they are more affordable than animal source foods in

\footnotetext{
*To whom correspondence should be addressed.

E-mail: aljawaldeha@who.int
}

the Eastern Mediterranean Region (EMR). Animal products may be widely available in the region, but their high cost makes it challenging for low-income populations to consume sufficient amounts of preformed vitamin A (1). Most countries in the EMR region, are classified as low- to middle-income and their populations showed a relatively low vitamin A intakes and food sources diversity.

Young children and pregnant women are the most vulnerable to vitamin A deficiency (VAD). VAD is the main cause of preventable maternal and childhood blindness and increases the risk of mortality from common childhood diseases such as diarrhea (2). In 2013, it was estimated that $29 \%$ of children $<5 \mathrm{y}$ of age in low- and middle-income countries globally were vitamin A deficient (3). The prevalence of VAD varies between countries within the EMR with several being classified as having VAD of severe public health significance, defined as a $\geq 20 \%$ prevalence of serum retinol concentrations $<0.70 \mu \mathrm{mol} / \mathrm{L}$ or $\geq 5 \%$ prevalence of 


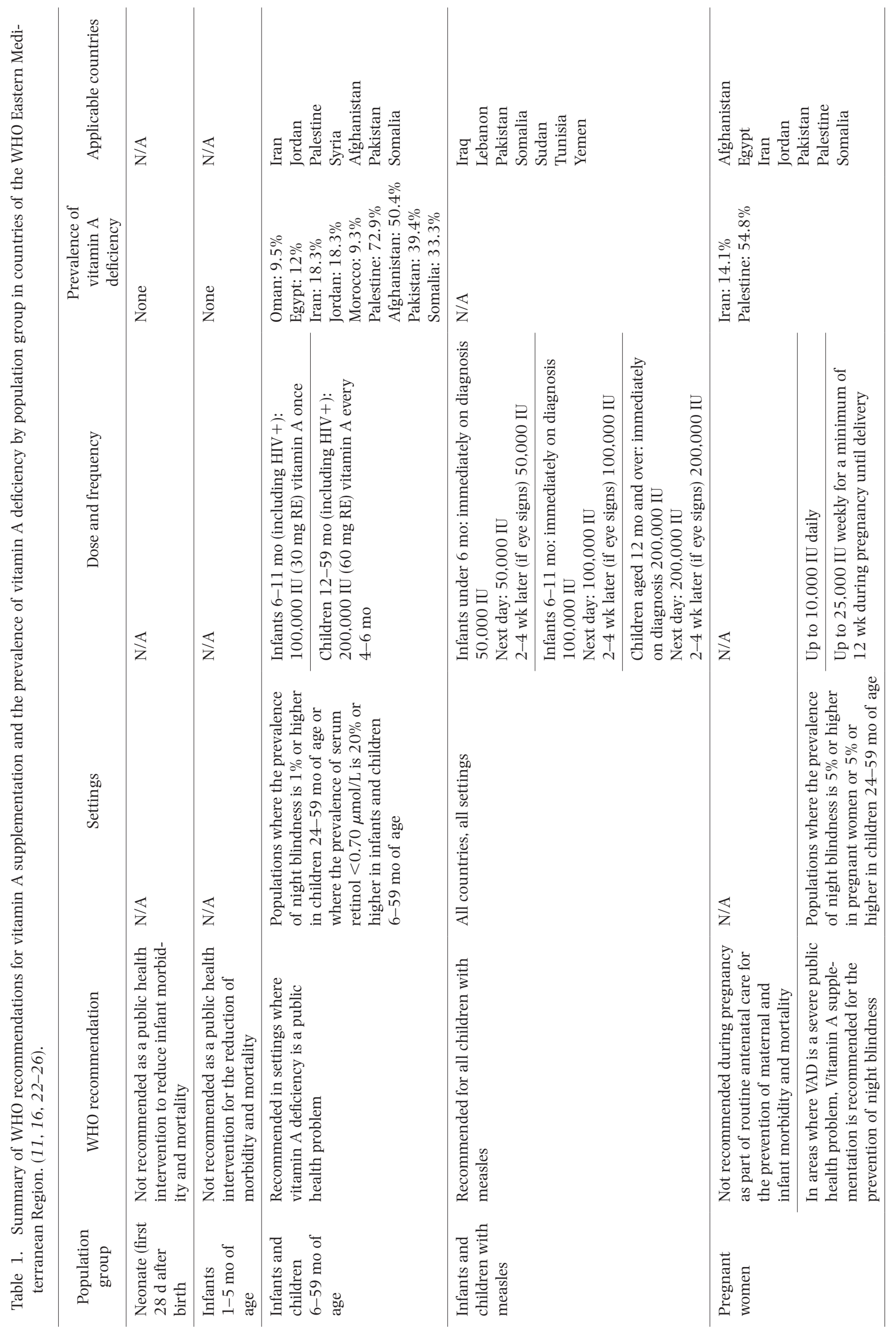


night blindness (4). Between 2004 and 2007, the prevalence of sub-clinical vitamin A deficiency was reported to be 10\% in Egypt and the Syrian Arab Republic, 17\% in Jordan, 20\% in Morocco, 20-30\% in Oman, and over $60 \%$ in Yemen, but only $2.3 \%$ in Tunisia (4). Between 2011 and 2015, the prevalence of $\operatorname{VAD}(<0.70 \mu \mathrm{mol} / \mathrm{L}$ retinol) among children $15-23$ mo of age and $14 \%$ in pregnant women in Iran (5), 50\% $<0.70 \mu \mathrm{mol} / \mathrm{L}$ retinol among children 6-59 mo in Afghanistan in 2013 (6), and $56 \%<0.70 \mu \mathrm{mol} / \mathrm{L}$ retinol in children 0-59 mo and $43 \%$ in women of reproductive age in Pakistan in 2011 (7).

There are several interventions being implemented in areas where vitamin A deficiency is of concern. High dose vitamin A supplementation is currently one of the most widely implemented vitamin A interventions in low- and middle-income countries. It is estimated that $62 \%$ of children $6-59$ mo of age in the 82 high priority countries targeted by UNICEF received the recommended two doses of high dose vitamin A supplementation in 2017 (8). Since the early 1990's, high dose vitamin A supplementation has been recommended by WHO for children 6-59 mo of age in settings where VAD is a public health problem for the reduction of morbidity and mortality related to preventable childhood diseases $(9,10)$. However, a more recent large study of high dose vitamin A supplementation in preschool age children in India (11) did not find a significant effect on child morbidity and mortality and some feel this intervention is no longer relevant due to reductions in the prevalence of diarrhea and diarrhea and their associated morbidity and mortality (12). An updated Cochrane systematic review of vitamin A supplementation for the prevention of morbidity and mortality in children from 6-59 mo of age reported a lower, but still significant, reduction in all-cause mortality [risk ratio (RR) 0.88; 95\% confidence interval (CI) 0.83 to 0.93 ], mortality due to diarrhea (RR 0.88 ; $95 \%$ CI 0.79 to 0.98 ), diarrhea incidence (rate ratio $0.85 ; 95 \%$ CI 0.82 to 0.87 ), measles incidence (rate ratio $0.50 ; 95 \%$ CI 0.37 to 0.67), incidence of Bitots spots (RR 0.42; 95\% CI 0.33 to 0.53) and incidence of night blindness (RR 0.32; 95\% CI 0.21 to 0.50) (13). As of 2011, WHO no longer recommends the use of high dose vitamin A supplementation for postpartum women or infants $1-5 \mathrm{mo}$ of age $(14,15)$.

High-dose vitamin A supplementation has contributed to reducing child mortality rates in low- and middle-income countries but does not address the underlying problem of inadequate vitamin A intakes. Nutrition interventions such as the fortification of staple foods with vitamin $A$ and the use of multiple micronutrient powders containing vitamin $\mathrm{A}$ are recommended in these settings $(16,17)$. Because the prevalence of VAD varies greatly with the EMR and is still a problem of public health significance in some countries, decisions to scale back or shift from universal high dose vitamin A supplementation should be based on information that verifies that vulnerable populations have adequate vitamin A status and access to sufficient dietary sources of 


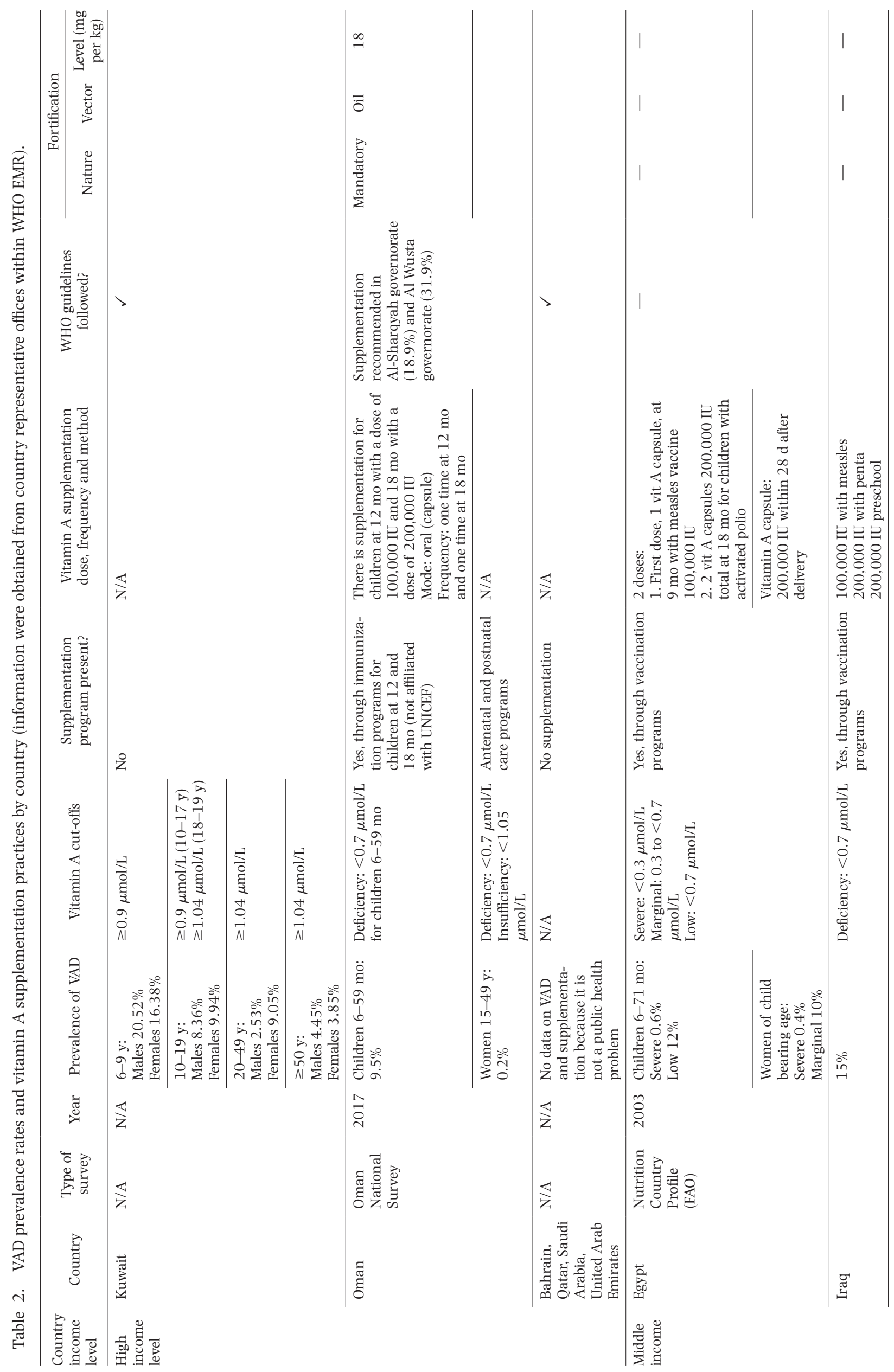


Vitamin A Status in EMR

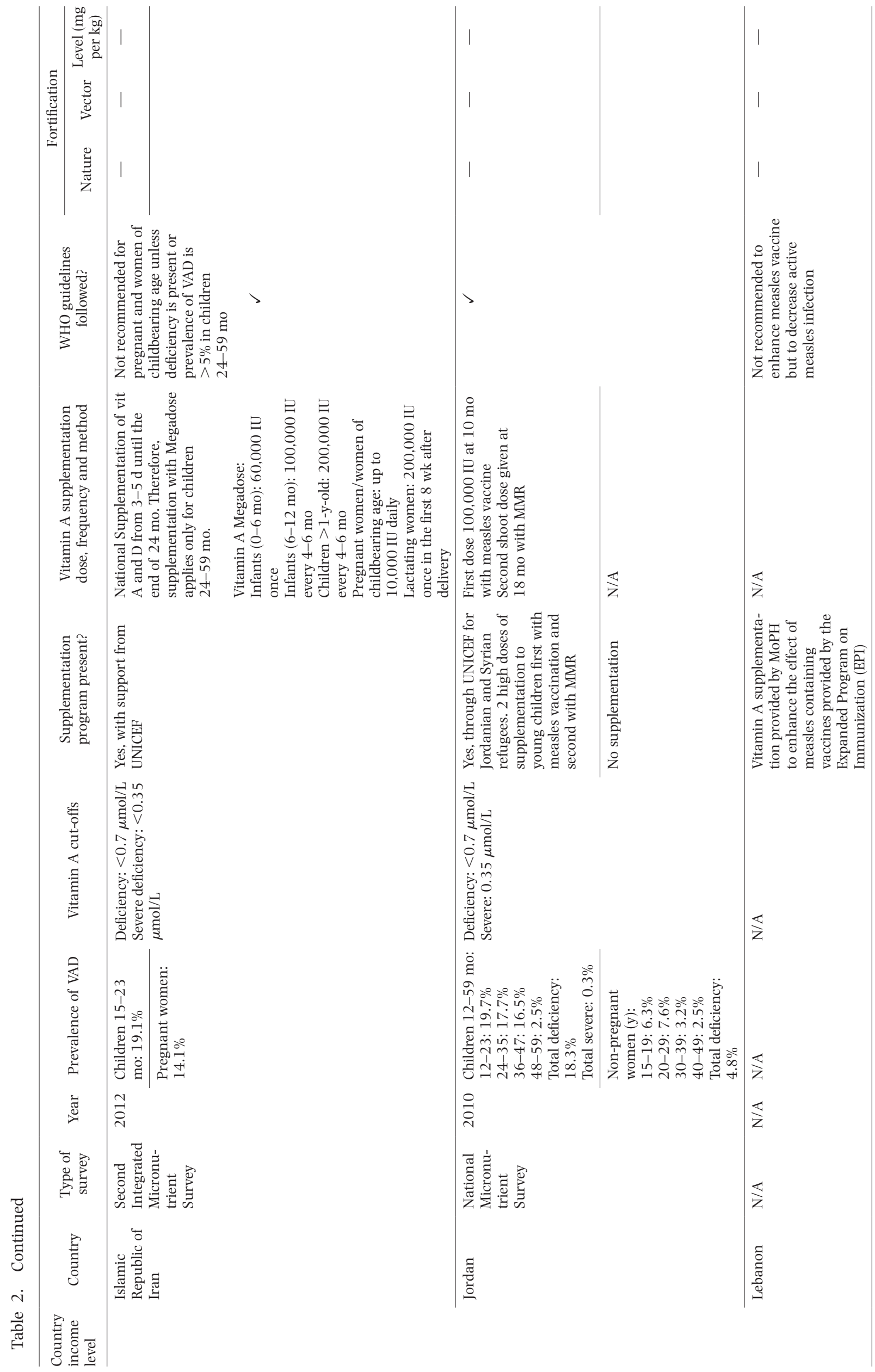




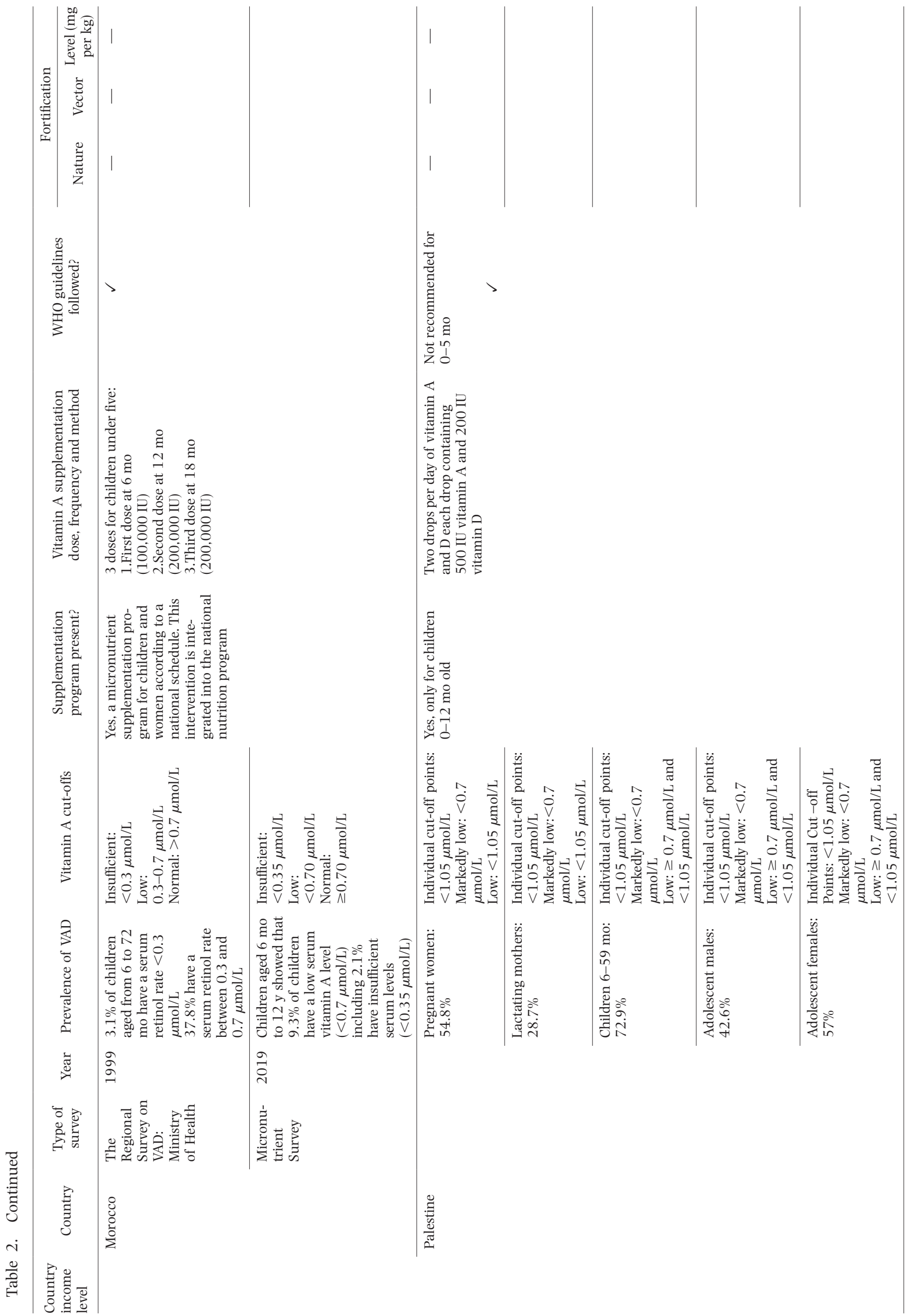




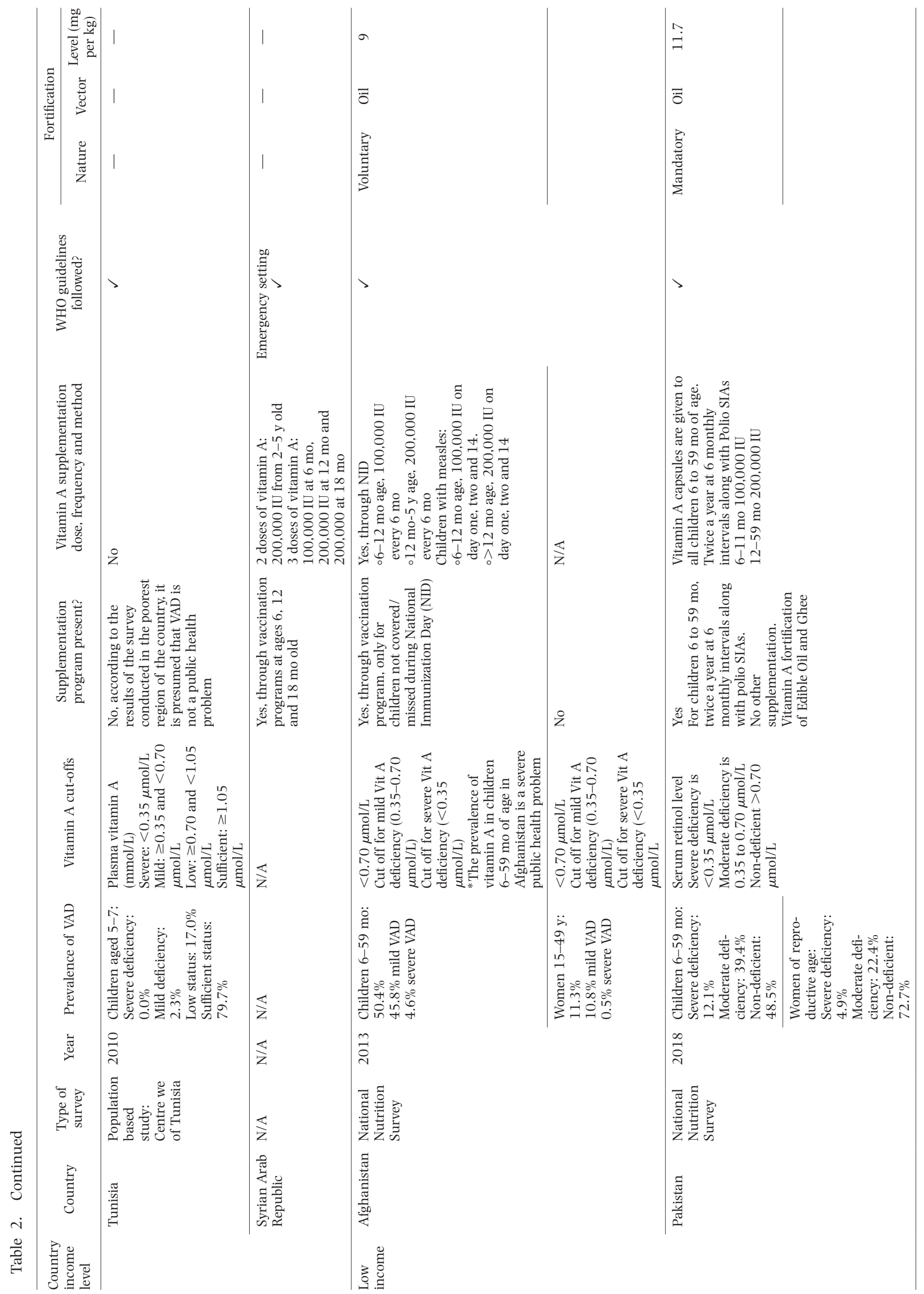




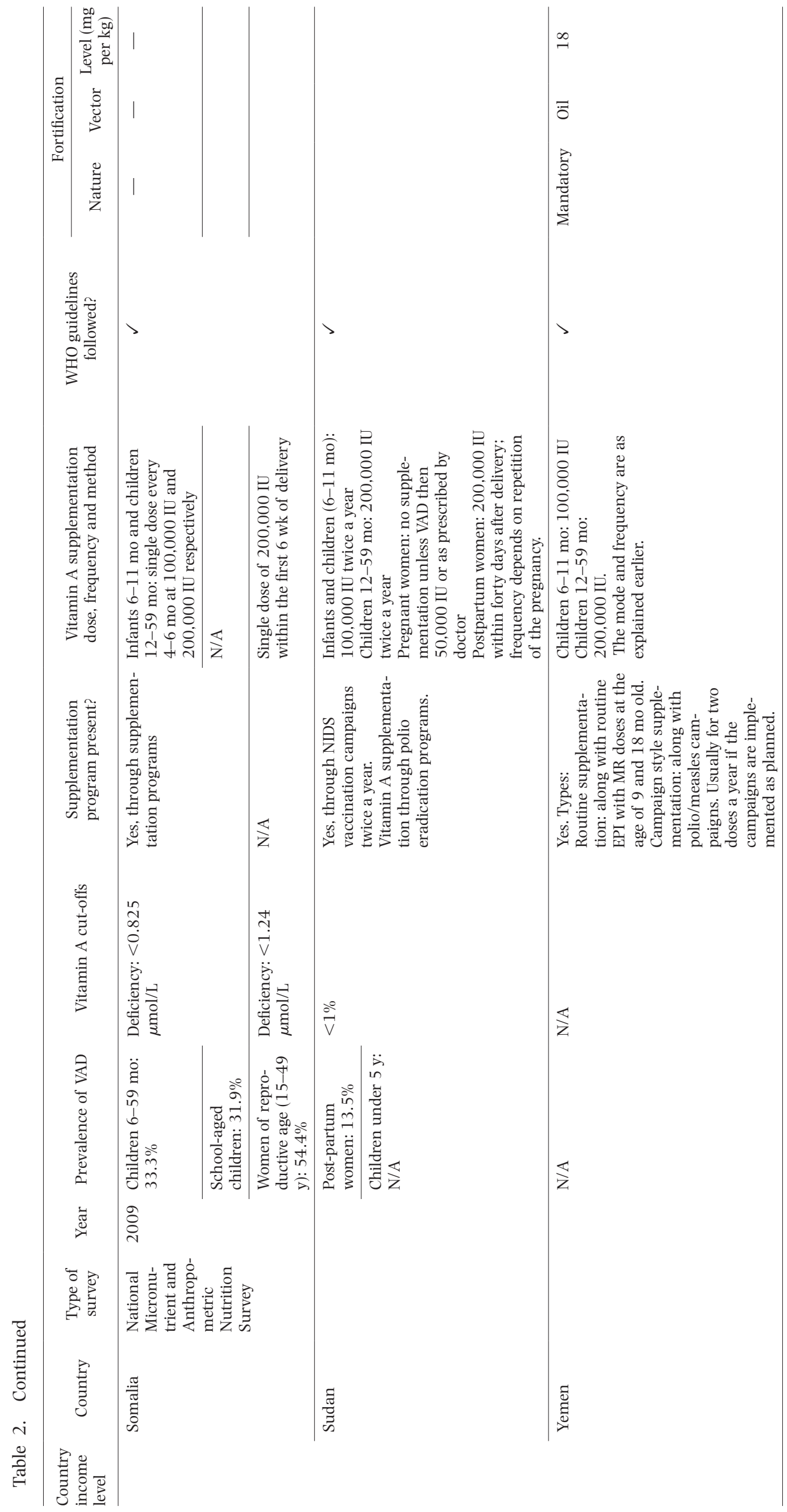


vitamin A. The Global Alliance for Vitamin A (18) has developed a process for helping countries to gather and evaluate the necessary evidence to aid countries in adapting the WHO guidelines on high dose vitamin A supplementation in infants and children 6-59 mo of age.

The aim of this paper is to review the WHO guidelines on vitamin A interventions and assess the current vitamin A supplementation practices across the EMR. As supplementation practices and the prevalence of VAD across the region is inconsistent, this review highlights that high dose supplementation is being provided to populations where VAD is not of public health concern. Gaps in supplementation practices are also identified to improve programmes within the region in compliance with WHO recommendations.

\section{Methodology}

The most current WHO guidelines for vitamin A supplementation in different age groups were retrieved from the WHO e-Library of Evidence of Nutrition Actions (19). A literature search was conducted to identify the most recent national nutrition surveys, as well as publications from UNICEF and the United Nations Food and Agriculture Organization (FAO) describing vitamin A supplementation practices in countries within WHO EMR. In addition, data on the vitamin A status of populations was retrieved using the Micronutrients Database in the WHO Vitamin and Mineral Nutrition Information System (20). Representatives from all 22 countries within WHO EMR were contacted to complete a questionnaire regarding the vitamin $\mathrm{A}$ status of their population, whether or not vitamin A supplementation programmes were being implemented, and if so, at what the dose, frequency and method of supplementation.

Countries within WHO EMR were classified by income. The categories include low, middle- and high-income countries. Afghanistan, Djibouti, Libya, Pakistan, Somalia, Sudan and Yemen are categorized as low-income countries. The middle-income countries in the region include Egypt, Iraq, Islamic Republic of Iran, Jordan, Lebanon, Morocco, Palestine, Syrian Arab Republic and Tunisia. High-income countries include Bahrain, Kuwait, Oman, Qatar, Saudi Arabia and United Arab Emirates.

\section{Results}

The most recent guidance on vitamin A supplementation for different population groups was published in 2011. Recommendations are available by the following life stages: neonate (first $28 \mathrm{~d}$ of life), infants aged 1-5 mo, infants and children aged 6-59 mo, pregnant women, pregnant women living with Human Immunodeficiency Virus (HIV), postpartum women, and infants and children with measles. Table 1 (10, 15, 21-25) summarizes the recommendations based on age group, settings, dose and frequency of supplementation and route of administration. In addition, the prevalence of VAD in each country in the EMR is added to each age group with a column of the countries that are recommended to supplement with vitamin A.

Information from 20 out of the 22 countries was available and the practices were compared with the WHO guidelines to determine if countries were following the recommended guidelines.

Countries across the EMR have revealed different supplementation practices depending on the income level of the country, the availability of vitamin A and the prevalence rates of VAD (Table 2) (e.g. Islamic Republic of Iran (26)). Although some countries had higher supplementation rates than others, the concern lies in the middle-income countries and their supplementation practices. Some of the countries across the region do not comply with the WHO guidelines for vitamin A supplementation for all recommended age groups. Throughout the analysis of the data, it was witnessed that the cut-off points used across countries within the EMR are different. The majority diagnosed deficiency when the serum retinol levels were $<0.7 \mu \mathrm{mol} / \mathrm{L}$, marginal when levels were between 0.3 to $<0.7 \mu \mathrm{mol} / \mathrm{L}$, and classified as severely deficient when levels were $<0.3 \mu \mathrm{mol} / \mathrm{L}$. However, countries including Kuwait, Palestine and Somalia, diagnosed deficiency when vitamin A levels were $<1.05 \mu \mathrm{mol} / \mathrm{L}$. This can create a differentiation between the data related to prevalence rates between countries since rates could be lower if different cut-offs are used.

High-income countries have little or no data related to VAD or supplementation practices because VAD is not a public health problem or non-existent in countries like Bahrain, Qatar, Saudi Arabia and United Arab Emirates. In this case, no supplementation programs are present in these countries. Kuwait has available data on the prevalence of VAD between the ages 6-50 y old in males and females. The prevalence among 10-19, $20-49$, and $\geq 50$ y old is minor with all rates $<10 \%$. The only age group that had higher levels of deficiency was 6-9 y old where the prevalence of VAD in males is $20.25 \%$ and $16.38 \%$ in females. However, since it is not considered a public health problem, Kuwait does not have a supplementation program present meaning they are following the recommended WHO guidelines.

Oman is the only high-income country that raises questions when it comes to their vitamin A supplementation practices. As a high-income country with access to high-quality diets rich in vitamin A foods, the prevalence among children ages 6-59 mo (9.5\%) and women $15-49$ y old $(0.2 \%)$ which is relatively low. Although rates are low, Oman supplements children at ages 12 (100,000 IU) and 18 (200,000 IU) mo through immunization programs, and women through antenatal and postnatal programs. This does not comply with the WHO guidelines since the prevalence of VAD among children is not $\geq 20 \%$. After taking a deeper look into the current situation in Oman, it has been noticed that governorates like Al-Sharqyah and Al Wusta have high rates of deficiency with rates of $18.9 \%$ and $31.9 \%$, respectively.

Unlike high-income countries in the EMR, the mid- 
dle-income countries face the greatest discrepancies in the rates of VAD and their supplementation practices. In most middle-income countries including Egypt, Islamic Republic of Iran, Jordan and Tunisia, VAD is considered to be a mild-to-moderate public health problem. However, in Palestine and Morocco, VAD is classified as a severe public health problem with prevalence rates of $72.9 \%$ and $37.8 \%$ among children $6-59$ mo, respectively. The prevalence of VAD in Egypt is $12 \%$ for children 6-71 mo and are being supplemented with vitamin $A$ at 9 mo when the measles vaccination is introduced, and then a second dose at 18 mo only for children with activated polio. However, there is no evidence that correlates vitamin A supplementation with treating polio. Since the 1990s vitamin A supplementation has been linked with polio National Immunization Days (NIDs), as a vehicle to reach children (27). This reveals that Egypt is following older recommendations that link vitamin A supplementation with decreasing morbidity and mortality rate among children. Although the Islamic Republic of Iran (prevalence 19.1\%) is following the recommended guidelines for children (15$23 \mathrm{mo}$ ), they are supplementing pregnant women and women of childbearing age with $10,000 \mathrm{IU}$ of vitamin A daily. This is not recommended unless there is a deficiency present or night blindness of children ages $24-59 \mathrm{mo}$ is $>5 \%$. Another country that is following older recommendations related to decreasing morbidity and mortality is Lebanon. Although there is no available data on the prevalence rates, Lebanon revealed that vitamin A supplementation is provided to enhance the effect of measles containing vaccines. However, this is not a recommendation in the WHO guidelines unless a child has an active measles infection. Other countries like Jordan, Morocco and Syria are implementing the WHO recommended guidelines properly by following the recommended dosage and setting.

Low-income countries have revealed the highest rates of VAD related to moderate acute or severe acute malnutrition. Due to higher rates of VAD, low-income countries including Afghanistan (children 6-59 mo: $50.4 \%$ ), Pakistan (39.4\%), Somalia (33.3\%) and Sudan VAD is considered a severe public health problem that needs solutions to prevent night blindness in vulnerable populations. All countries have supplementation programs in place that comply with the WHO guidelines. In addition, with higher VAD rates among pregnant women and women of child bearing age, supplementation is provided to mothers within the first $40 \mathrm{~d}$ after delivery as recommended by WHO in Somalia and Sudan.

\section{Discussion}

Although some high-to-middle income countries across the EMR consume relatively healthy diets, VAD remains a burden on most low-income countries and a few middle-income countries. Despite the burden and the efforts made to supplement with vitamin A in order to improve the status of those most vulnerable, countries continue to misinterpret the recommended guide- lines suggested by WHO. Previously, countries were advised to supplement with vitamin A to reduce morbidity and mortality rates among infants, children and pregnant women. UNICEF collaborated with several immunization programs to provide two high doses of vitamin A. By taking this initiative, it increased the coverage rates in low-income countries. Low-income countries need access to vitamin A supplements. Although a lot of progress has been made over the past two decades, supplementation has recently become more difficult. This is because as the world works to eradicate polio, many countries have discontinued the implementation of polio immunization programmes. In low-income countries, these programmes are the vehicle for delivery of vitamin A supplements (28).

Since supplementation is not always available in low-income countries, it is important to consider other methods of preventing and treating VAD. One of the most common and efficient methods includes fortification of staple foods. Vitamin A is one of many vitamins and minerals that is added to staple foods including vegetable oil, wheat flour, rice, sugar and many more. At the time of study only 3 countries (Afghanistan, Pakistan and Yemen) have issued standards for the fortification of oil with vitamin A. Low compliance was reported in Afghanistan as low as 30\% while it reaches $68 \%$ in Pakistan. Wheat flour could be fortified with vitamin A since it is stable in flour and does not affect the smell, taste and appearance of the flour. However, throughout the preparation of wheat flour, the vitamin A content could be affected due to high humidity and temperatures (29). WHO has constructed recommendations on the average levels of nutrients to add to wheat flour based on extraction, fortification compound and per capita flour availability (30). It is recommended to fortify with vitamin A palmitate at different levels based on average per capita wheat flour availability. The recommendations are based on population at risk of VAD. However, since vegetable oil is highly consumed in the EMR, fortification of vegetable oil with vitamin A would be the most cost-effective technique to aid in the improvement of vitamin A status.

In addition to fortification, efforts should be made to increase awareness on the importance of consuming a balanced and healthy diet that includes the intake of fruits, vegetables, protein, carbohydrates and fats. A healthy lifestyle starts off with breastfeeding. Mothers in low, middle- and high-income countries should be encouraged to breastfeed to support the needs of their infants. Breast milk is a rich source of vitamin A that adjusts to the amount an infant requires.

The current vitamin A situation and trend during the last three decades vary across the WHO regions. Indeed, the most affected ones by VAD are the African Region (47\% (95\% CI: 24-73) in 2013) and South East Asian Region (36\% (95\% CI: 9-67) in 2013) (3). The noticeable reduction in VAD was denoted in the Western Pacific Region from 40\% (95\% CI: 14-73) in 1991 to 6\% (95\% CI: 1-6) in 2013 while prevalence still consistently the same for the Eastern Mediterranean Region 
over the period of 1991-2013 or increased slightly in African Region (+4\%) (3). The Regions of Americas VAD prevalence decreased by 10\% since 1991 to reach 11\% (95\% CI: 4-23) in 2013. In 2017, the number of Disability-Adjusted Life Years (DALYs) reach more than one million in two regions, namely Africa Region and South-East Asia Region (Global Health Data Exchange, http://ghdx.healthdata.org/). However, the number of DALYs declined over the last three decades.

Some limitations to this study include the difference in the cut-off points of serum retinol used to identify VAD. With countries using different cut-offs it makes it difficult to compare VAD across the region. Some rates could potentially be higher or lower depending on the cut-offs used. In addition, the data on VAD has not been updated in all countries within the region. Each country has information from different years, which could be another limitation when analyzing the data. The available data across the EMR varies in the age groups studied in countries. Most countries have studied infants and children ages 6-59 mo, pregnant women and women of childbearing age. However, some countries only have data available on older children and adolescents. This limits the analysis of the results as two age groups cannot be compared to one another.

In conclusion, to support Member States to appropriately follow and implement the WHO guidelines for vitamin A supplementation, there are a few recommendations to help the EMR. After investigating the current situation of VAD and supplementation practices in the region recommendations for countries with different income levels differ.

For high income countries, it is not recommended to supplement with vitamin A since deficiency is extremely low or non-existent. However, in countries like Oman where VAD is not a public health problem but concerning levels of VAD are present in some governorates where nomads reside, it is recommended to only implement supplementation programs that comply with the WHO guidelines in Al-Sharqyah and Al Wusta.

The first step-in middle-income countries to change current practices is to identify if the country needs a supplementation program or not by looking at the prevalence of deficiency and matching it with the WHO recommendations. There is a need to re-evaluate the target population that focus on targeted populations where VAD is a public health concern. Since the highest rates of deficiency are among children 6-59 mo, middle income countries should focus on only supplementing children in populations where the prevalence of night blindness is $\geq 1 \%$ in children $24-59$ mo of age or where the prevalence of VAD is $\geq 20 \%$ in infants and children $6-59 \mathrm{mo}$ of age, using the cut-off $<0.7 \mu \mathrm{mol} / \mathrm{L}$. Dose, frequency and method should also comply with WHO guidelines. This applies to Eastern Mediterranean countries including Iran, Jordan, Morocco, Palestine and Syria.

As for low income countries, since all Eastern Mediterranean countries under this category comply with the WHO guidelines, it is recommended to continue to supplement with vitamin A in parallel with the recommendations. If any improvements take place in low-income countries, it is recommended to re-evaluate supplementation practices to determine if supplementation is still needed in specific age groups or governorates.

As an overall recommendation for all countries in the EMR, countries should work towards increasing awareness on consuming a healthy diet rich in various vitamins and mineral including vitamin A rich foods. In addition, it is highly recommended to improve the status of vitamin A through fortification programs focused on fortifying wheat flour, rice or vegetable oil.

\section{Authorship}

Review concept and design: AA and FS, interpretation: FS, LR, RD and AA, writing manuscript: FS, RD, LR and AA.

\section{Disclosure of state of COI}

No conflicts of interest to be declared.

\section{Acknowledgments}

This review is funded by the Eastern Mediterranean Regional Office of the World Health Organisation.

\section{REFERENCES}

1) WHO. 2004. Vitamin and mineral requirements in human nutrition. World Health Organization and Food and Agriculture Organization of the United Nations.

2) UNICEF. 2020. UNICEF global databases, based on administrative reports from countries. Vitamin A supplementation coverage 2000-2018 for current priority country list.

3) Stevens GA, Bennett JE, Hennocq Q, Lu Y, De-Regil LM, Rogers L, Danaei G, Li G, White RA, Flaxman SR, Oehrle SP, Finucane MM, Guerrero R, Bhutta ZA, Then-Paulino A, Fawzi W, Black RE, Ezzati M. 2015. Trends and mortality effects of vitamin A deficiency in children in 138 low-income and middle-income countries between 1991 and 2013: a pooled analysis of population-based surveys. Lancet Glob Health 3: e528-e536.

4) Bagchi K. 2008. Nutrition in the eastern Mediterranean region of the World Health Organization. East Mediterr Health J 14: S107-S113.

5) Pouraram H, Djazayery A, Mohammad K, Parsaeian M, Abdollahi Z, Motlagh AD, Djalali M, Khodaverdian K, Sotoudeh G, Yarparvar A, Heshmat R, Siassi F. 2018. Second national integrated micronutrient survey in Iran: Study design and preliminary findings. Arch Iran Med 21: 137.

6) Ministry of Public Health (Afghanistan), UNICEF. 2014. National Nutrition Survey Afghanistan 2013. Ministry of Public Health, Kabul.

7) Government of Pakistan. 2013. National Nutrition Survey 2011. Planning and Development Division, Government of Pakistan, Islamabad.

8) UNICEF. 2019. The State of the World's Children 2019. Children, Food and Nutrition: Growing well in a changing world. UNICEF, New York.

9) WHO/UNICEF/IVACG Task Force. 1997. Vitamin A supplements: a guide to their use in the treatment and prevention of vitamin A deficiency and xerophthalmia. World Health Organization, Geneva. 
10) WHO. 2011. Guideline: vitamin A supplementation in infants and children 6-59 months of age. World Health Organization, Geneva.

11) Awasthi S, Peto R, Read S, Clark S, Pande V, Bundy D, DEVTA (Deworming and Enhanced Vitamin A) Team. 2013. Vitamin A supplementation every 6 months with retinol in 1 million pre-school children in north India: DEVTA, a cluster-randomised trial. Lancet 381: 14691477.

12) Mason JB, Sanders D, Greiner T, Shrimpton R, Yukich J. 2016. Vitamin A deficiency: policy implications of estimates of trends and mortality in children. Lancet Glob Health 4: e21.

13) Imdad A, Mayo-Wilson E, Herzer K, Bhutta ZA. 2017. Vitamin A supplementation for preventing morbidity and mortality in children from six months to five years of age. Cochrane Database Syst Rev 3: Cd008524.

14) Oliveira-Menegozzo JM, Bergamaschi DP, Middleton P, East CE. 2010. Vitamin A supplementation for postpartum women. Cochrane Database Syst Rev 10: CD005944.

15) WHO. 2011. Vitamin A supplementation for infants 1-5 months of age. World Health Organization, Geneva.

16) WHO. 2019. Multiple micronutrient powders for pointof-use fortification of foods consumed by children 2-12 years of age. World Health Organization [update February 11th, 2019].

17) WHO. 2019. Multiple micronutrient powders for pointof-use fortification of foods consumed by children 6-23 months of age. World Health Organization [update February 11th, 2019].

18) The Global Alliance for Vitamin A. 2019. Conditions for scaling back universal preschool vitamin A supplementation. Policy Brief.

19) Khan WU, Sellen DW. 2011. e-Library of Evidence for
Nutrition Actions (eLENA). World Health Organization, Geneva.

20) WHO. 2011. Vitamin and Mineral Nutrition Information System (VMNIS).

21) WHO. 2011. Guideline: Neonatal vitamin A supplementation. World Helath Organization, Geneva. p 1-9.

22) WHO. 2019. Essential nutrition actions: mainstreaming nutrition through the life-course. World Health Organization, Geneva.

23) WHO. 2011. Guideline: vitamin A supplementation in pregnant women. World Health Organization, Geneva.

24) WHO. 2011. Vitamin A supplementation in pregnancy for reducing the risk of mother-to-child transmission of HIV. World Health Organization, Geneva.

25) WHO. 2011. Vitamin A supplementation in postpartum women. World Health Organization, Geneva.

26) Pouraram H, Djazayery A, Mohammad K, Parsaeian M, Abdollahi Z, Dorosty Motlagh A, Djalali M, Khodaverdian K, Sotoudeh G, Yarparvar A, Heshmat R, Siassi F. 2018. Second National Integrated Micronutrient Survey in Iran: Study design and preliminary findings. Arch Iran Med 21: 137-144.

27) UNICEF. 2007. Vitamin A Supplementation: A decade of progress. The United Nations Children's Fund (UNICEF).

28) The Lancet. 2018. Vitamin A distribution in danger. Lancet 391: 1866.

29) WHO. 2009. Recommendations on wheat and maize flour fortification meeting report: Interim consensus statement. World Health Organization, Geneva.

30) Al Jawaldeh A, Pena-Rosas JP, McColl K, Johnson Q, Elmadfa I, Nasreddine L. 2019. Wheat flour fortification in the Eastern Mediterranean Region. WHO Regional Office for the Eastern Mediterranean, Cairo. 